\title{
Sistema agroforestal Quesungual como fuente natural de emisiones de gases de efecto invernadero en la microcuenca Tecomapa, Somotillo-Nicaragua
}

\section{Quesungual agroforestry system as a natural source of greenhouse gas emissions in the Tecomapa micro- basin, Somotillo-Nicaragua}

\author{
Beatriz Acuña ${ }^{1}$, Sandra Loaiza ${ }^{2}$, Reynaldo B. Mendoza Corrales ${ }^{3}$, Ngonidzashe Chirinda ${ }^{4}$ \\ ${ }^{1}$ Universidad Nacional Agraria, Managua, Nicaragua / ORCID: https://orcid.org/0000-0002-3754-8112 \\ ${ }^{2}$ Centro Internacional de Agricultura Tropical, AA 6713 Cali, Colombia / ORCID: https://orcid.org/0000-0002-0239-896X \\ ${ }^{3}$ Universidad Nacional Agraria, Managua Nicaragua / ORCID: https://orcid.org/0000-0003-4382-798v \\ ${ }^{4}$ Mohammed VI Polytechnic University, Marruecos / ORCID: https://orcid.org/0000-0002-4213-6294
}

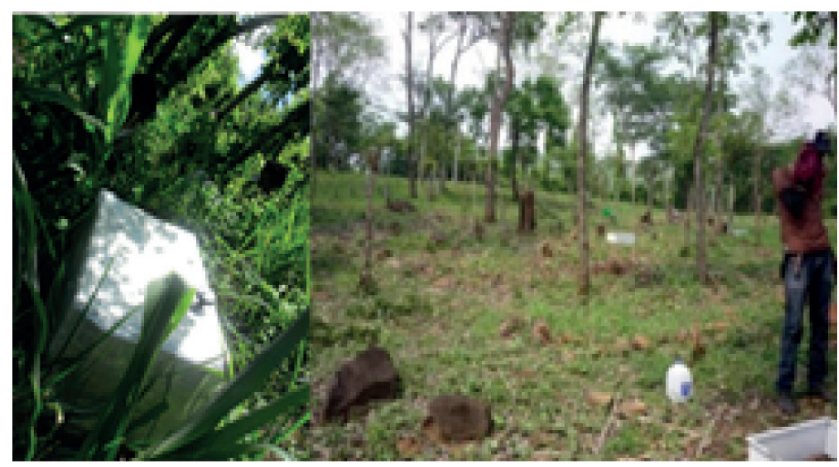

\section{RESUMEN}

Con el propósito de evaluar las emisiones de metano y óxido nitroso en el suelo a través del método de cámaras estáticas cerradas en tres sistemas de uso; bosque secundario, sistema agroforestales Quesungual y sistema tradicional agrícola, en la micro cuenca Tecomapa, Somotillo, Nicaragua, setenta y dos cámaras estáticas cerradas fueron establecidas en seis fincas localizadas en la unidad hidrográfica Tecomapa, Nicaragua en 2014, para evaluar las emisiones de metano $\left(\mathrm{CH}_{4}\right)$ y óxido nitroso $\left(\mathrm{N}_{2} \mathrm{O}\right)$, en los sistemas de uso de suelo: Bosque secundario, Quesungual y Sistema Tradicional agrícola. Como resultado, mayores emisiones de $\mathrm{N}_{2} \mathrm{O}$ y $\mathrm{CH}_{4}$, se observaron en el mes más lluvioso (septiembre 2014). El sistema Quesungual fue sumideros de $\mathrm{CH}_{4}$ a nivel de flujos netos acumulados ( $p>\mathrm{F}=0.0133)$, y el sistema tradicional el mayor emisor de este gas. La mayor emisión de $\mathrm{N}_{2} \mathrm{O}$ tiende a ocurrir en el sistema tradicional. Finalmente el gas metano se asoció positivamente al $\mathrm{N}_{2} \mathrm{O}$ $\left(\mathrm{r}^{2}=1 ; p>\mathrm{F}=0.0533\right)$, y el WFPS tiende a asociarse negativamente a la temperatura y gases $\mathrm{CH}_{4}$ y $\mathrm{N}_{2} \mathrm{O}$.

Palabras clave: metano, óxido nitroso, cambio climático.
ABSTRACT
In order to evaluate the emissions of methane and nitrous oxide in the soil through the closed static chamber method in three systems of use; secondary forest, Quesungual agroforestry system and traditional agricultural system, in the Tecomapa micro basin, Somotillo, Nicaragua, seventy-two closed static chambers were established in six farms located in the Tecomapa hydrographic unit, Nicaragua in 2014, to evaluate the emissions of methane $\left(\mathrm{CH}_{4}\right)$ and nitrous oxide $\left(\mathrm{N}_{2} \mathrm{O}\right)$, in the land use systems: Secondary forest, Quesungual and Traditional agricultural systems. As a result, higher $\mathrm{N}_{2} \mathrm{O}$ and $\mathrm{CH}_{4}$ emissions were observed in the rainiest month (September 2014). The Quesungual system was $\mathrm{CH}_{4}$ sinks at the level of accumulated net flows $(p>\mathrm{F}=0.0133)$, and the traditional system was the largest emitter of this gas. The greatest emission of $\mathrm{N}_{2} \mathrm{O}$ tends to occur in the traditional system. Finally, methane gas was positively associated with $\mathrm{N} 2 \mathrm{O}\left(\mathrm{r}^{2}=1 ; p>\mathrm{F}=0.0533\right)$, and WFPS tends to be negatively associated with temperature and $\mathrm{CH}_{4}$ and $\mathrm{N}_{2} \mathrm{O}$ gases.

Keywords: Methane, nitrous oxide, climate change.

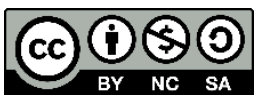

(C) Copyright 2020. Universidad Nacional Agraria (UNA).
Los artículos de la revista La Calera de la Universidad Nacional Agraria, Nicaragua, se comparten bajo términos de la licencia Creative Commons: Reconocimiento, No Comercial, Compartir Igual. Las autorizaciones adicionales a las aquí delimitadas se pueden obtener en el correo edgardo.jimenez@ci.una.edu.ni 


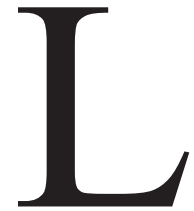

a conversión de sistemas de bosques nativos a sistemas pecuarios en las regiones del trópico contribuye al incremento de emisiones de gases de efecto invernadero en América Latina según la organización de las Naciones Unidas para la Alimentación y la Agricultura (FAO, 2019). En Nicaragua los resultados del Segundo Inventario Nacional de gases de efecto invernadero (GEI), revelan que Nicaragua pasó de ser fijador de gases a emisor como consecuencia de las practicas agronómicas de los sistema productivos y el cambio de uso de suelo forestal a agrícola y pecuario según el Programa de las Naciones Unidas para el Desarrollo (PNUD, 2008). Los sistemas agroforestales en las regiones de Honduras y Nicaragua son conocidos como sistema agroforestal Quesungual (SAQ) que se caracterizan por ser sistemas conservacionistas en el manejo de la regeneración natural en relación con la producción de cultivos anuales (maíz y frijol), y el manejo de árboles, por medio de las prácticas de la no quema y la siembra directa al espeque (palo con punta en el extremo para hacer el orificio de siembra) como estrategia de mitigación del cambio climático (Ferreira 2008; Nair, Nair, Kumar y Haile, 2009; Avilés, 2016).

A partir de técnicas de conservación del sistema agroforestal Quesungual (SAQ) como estrategia de mitigación y las pocas investigaciones en cuantificación de GEI que presenta la región de Nicaragua nace la idea de monitorear las emisiones de gases de efecto invernadero entre el sistema de bosque y los SAQ para determinar el impacto ambiental de estos sistemas característicos de Centro América. El presente estudio pretende evaluar las emisiones de metano y óxido nitroso en el suelo a través del método de cámaras estáticas cerradas en tres sistemas de uso; bosque secundario, sistema agroforestales Quesungual y sistema tradicional agrícola, en la comunidad La Danta y la Flor, unidad hidrográfica Tecomapa, Somotillo, Nicaragua. A la vez, identificar las relaciones entre las emisiones de gases de efecto invernadero con las condiciones climáticas de las zonas de estudio y las condiciones físico -químicas del suelo.

\section{MATERIALES Y MÉTODOS}

Descripción de la zona de estudio. La microcuenca Tecomapa, se localiza en el municipio de Somotillo, departamento de Chinandega, entre las coordenadas $13^{\circ} 1008^{\prime} .94^{\prime \prime}$ y $13^{\circ} 0643^{\prime} .92^{\prime \prime}$ latitud Norte y $86^{\circ} 50^{\prime} 54.93 "$ y $86^{\circ} 52^{\prime} 04.44^{\prime \prime}$ longitud Oeste. Elevación entre 136 y 400 $\mathrm{m}$. Suelos arcillosos friables, moderadamente profundos pertenecientes a los órdenes: Alfisoles (46\%), Molisoles (35.4\%), Vertisoles (15.3\%) y los Entisoles con (2.9\%) (Rodríguez, Calero, Avilés y Mendoza, 2008). El área se encuentra en la zona climática de Sabana según clasificación de Köppen, con una precipitación promedia anual bimodal entre $1400-1600 \mathrm{~mm}$ distribuido en seis meses de lluvias de mayo a noviembre, con temperaturas media anual entre 24 y $25^{\circ} \mathrm{C}$, Instituto Nicaragüense de Tecnología Agropecuaria (INTA), 2005; Instituto Nicaragüense de Estudios Territoriales (INETER 2014), y clasificada ecológicamente en la zona de vida Bosque tropical seco (Khatun, Imbach y Zamora, 2013) (Figura 1).

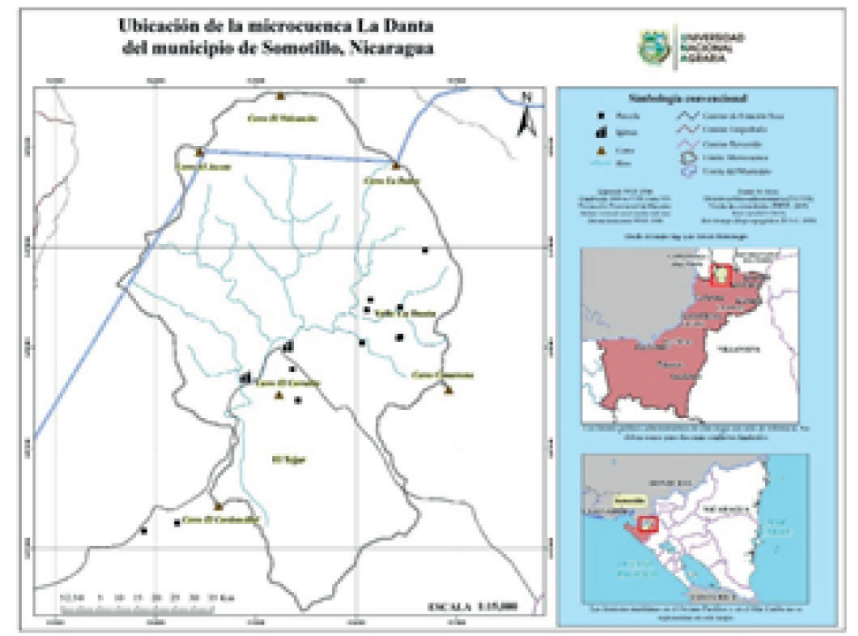

Figura 1. Localización geográfica de las zonas de estudio.

Diseño experimental. En seis fincas de productores localizadas en las comunidades La Danta y La Flor (microcuenca Tecomapa), fueron seleccionadas tres parcelas de usos aleatorio; Bosque secundario, Sistema Quesungual y el Sistema tradicional de siembra (Cuadro 1), donde fueron establecidas un sistema de cuatro cámaras cerradas para monitorear emisiones de gases. Cada finca representó una réplica, para un total de 72 cámaras estáticas de gases utilizadas para monitorear mensualmente. La fertilización nitrogenada fue aplicada en el cultivo de maíz de manera fraccionada a los 25 y 45 días después de siembra (dds), a una dosis total de $59.5 \mathrm{~kg} \mathrm{~N} \mathrm{ha}^{-1}$.

Para el análisis de datos se utilizó un diseño de Bloques Completo al Azar, donde las fincas se asignaron como estructura de bloques, las parcelas de uso como tratamientos aleatorios y las cámaras como unidades experimentales, donde se tomaba el dato de los gases y temperatura.

Monitoreo de gases. Las cámaras estáticas de gases se ubicaron en seis fincas de productores, con la presencia de tres tipos de uso: Bosque secundario, Sistema Quesungual y el Sistema tradicional de siembra (Cuadro 1).

Las fechas de muestreo fueron mensuales e iniciaron el 29 de mayo del 2014, y finalizaron el 15 de diciembre de ese mismo año. Muestreo excepcional e intensificado se realizó al momento de fertilizar los cultivos de maíz y frijol (tres días de monitoreo consecutivos después de la aplicación del fertilizante). 
Cuadro 1. Características de las fincas, La Danta, Somotillo, 2014

\begin{tabular}{|c|c|c|c|c|c|}
\hline \multirow[t]{2}{*}{ Productor (a) } & \multirow[t]{2}{*}{ Comunidad } & \multirow[t]{2}{*}{ Tipo de suelo } & \multirow{2}{*}{$\begin{array}{l}\text { Elevación } \\
\text { (m) }\end{array}$} & \multicolumn{2}{|c|}{ Localización } \\
\hline & & & & Norte & Oeste \\
\hline Teresa R & La Flor & Alfisol y Vertisol & 136 & $13^{\circ} 8.158$ & $86^{\circ} 52.313$ \\
\hline Juana $\mathrm{H}$ & La Flor & Alfisoles & 140 & $13^{\circ} 8.200$ & $86^{\circ} 52.130$ \\
\hline Isidro $\mathrm{Z}$ & La Danta & $\begin{array}{l}\text { Alfisol, Entisoles, y } \\
\text { Molisoles }\end{array}$ & $174-187$ & $13^{\circ} 9.032$ & $86^{\circ} 51.493$ \\
\hline $\begin{array}{l}\text { Gerónimo } \\
\text { Aguilera }\end{array}$ & La Danta & $\begin{array}{l}\text { Alfisoles, Entisoles y } \\
\text { Molisoles }\end{array}$ & 189 & $13^{\circ} 9.407$ & $86^{\circ} 51.064$ \\
\hline reddy A & La Danta & Alfisoles y Eentisoles & $169-236$ & $13^{\circ} 9.199$ & $86^{\circ} 50.905$ \\
\hline Roberto P & La Danta & Entisoles y Alfisoles & $318-400$ & $13^{\circ} 9.674$ & $86^{\circ} 50.761$ \\
\hline
\end{tabular}

Para instalar las cámaras de gases en campo, se colocaron bases rectangulares de aluminio distribuidas en las parcelas en forma de "Y". La parte inferior de la base tiene una altura de $20 \mathrm{~cm}$, de los cuales $10 \mathrm{~cm}$ son enterrados en el suelo. Al instalarse se dejaron aproximadamente dos meses antes de la toma de muestras de gases para estabilizar el sistema (Figura 2).

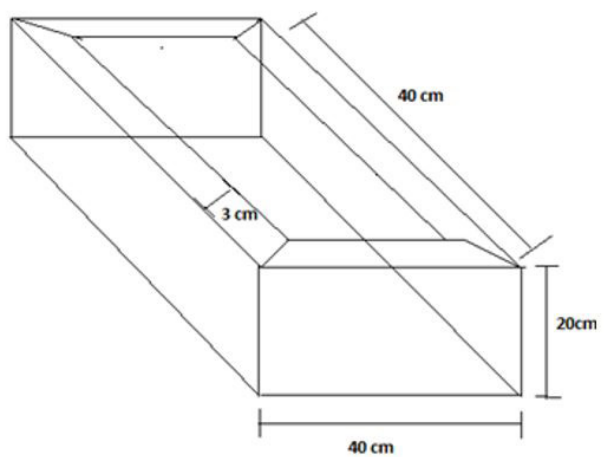

Figura 2. Base cámara estática cerrada. Proyecto CIAT-UNA GEI 2014.

Las muestras de gases fueron enviadas al laboratorio del CIAT para determinar concentraciones de $\mathrm{N}_{2} \mathrm{O}$ y $\mathrm{CH}_{4}$, utilizando un cromatógrafo de gases Shimadazu GC-14A con detectores FID y ECD.

Monitoreo de flujo de metano y óxido nitroso. Al momento de tomar las muestras se colocó una cámara de polipropilieno cubierta con un aislante de calor, la cual tiene un agujero con un septum de goma para monitorear la temperatura. En esta etapa se realizó la técnica del Gas Pooling (Arias-Navarro et al., 2015), donde una muestra de gas total compuesta por sub muestras obtenidas en intervalos de $0,10,20$ y 30 minutos a partir de la instalación de la cámara, finalmente el gas es depositado en viales que son enviados al laboratorio (Figura 3).

Las cámaras poseen un segundo agujero con una tapa de goma en la cual se colocó un termómetro digital y se tomaron las temperaturas dentro de la cámara cerrada. El termómetro digital siempre se colocó en la segunda cámara de cada parcela, tomando la temperatura en grados Celsius $\left({ }^{\circ} \mathrm{C}\right)$ tomada en similares intervalos, al gas total.
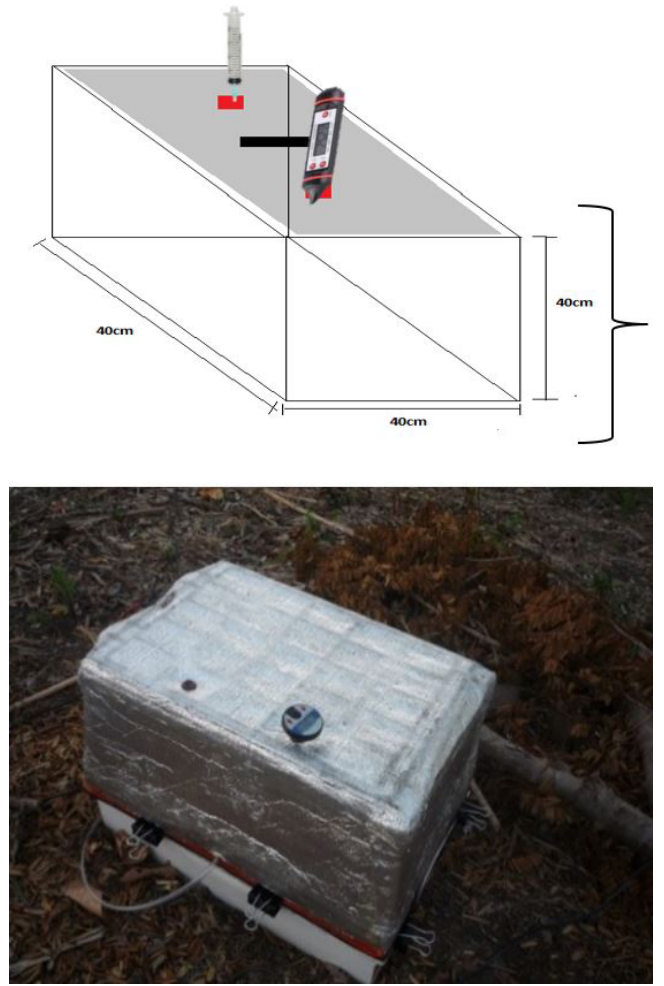

Figura 3. Parte superior: Cámara de polímeros con dos septum (redondos rojos) para la inserción de la jeringa y termómetro.

La fórmula que se usó para determinar los flujos diarios se describe a continuación.

$$
E=\frac{d N_{2} O}{d t}+\frac{V M}{A V m}
$$

Dónde:

E: Flujo de gas $\left(\mathrm{CH}_{4}, \mathrm{~N}_{2} \mathrm{O}\right.$ o $\left.\mathrm{CO}_{2}\right)$ por unidad de área y tiempo. $d N_{2} O$ : Rango de acumulación del gas en el aire dentro de $\frac{d N_{2}}{d t}$ la cámara expresado en partes por millón por minuto y $\mathrm{CH}_{4}$ ).

$\mathrm{V}$ : volumen de la cámara

A: área de la cámara

$\mathrm{M}$ : masa de $\mathrm{N}$ por mol de $\mathrm{N} 2 \mathrm{O}$

$\mathrm{Vm}$ : volumen molecular

El valor de Vm se calcula de la ecuación de gases ideales como se muestra a continuación,

$\mathrm{Vm}=\mathrm{nRT} / \mathrm{P}$ 
Dónde:

$\mathrm{Vm}=$ Volumen molecular de un gas

$\mathrm{n}=$ Moles, (en este caso necesitamos calcular el volumen de una (1) mol del gas).

$\mathrm{R}=$ constate de la ley de los gases ideales $(0.0820574587 \mathrm{~L}$ atm K $\mathrm{K}^{-1} \mathrm{~mol}^{-1}$ ).

$\mathrm{T}=$ Temperatura dentro de la cámara

Monitoreo de suelo. En el laboratorio de suelos y agua (LABSA) de la Universidad Nacional Agraria (UNA), localizada en Managua, se realizaron los análisis de humedad de suelo utilizando el método gravimétrico USDA, (1962) y los análisis químicos de nitrógeno total utilizando el método Kjeldahl $\mathrm{N}$, publicado por Bremner \& Mulvaney en 1982. Para el muestreo de suelo se utilizó un barreno de espiral, y se colectaron mensualmente una muestra compuesta de 0.5 $\mathrm{kg}$ proveniente de cuatro cámaras, a $10 \mathrm{~cm}$ de profundidad. Dicha extracción se hizo a un lado de las cámaras.

Análisis estadístico. Un diseño de Bloques Completo al Azar fue utilizado para analizar el comportamiento de cada gas por uso del suelo, finca y fechas de muestreo. Utilizando el siguiente modelo aditivo lineal:

$\mathrm{Y}_{\mathrm{ij}}=\mu_{\mathrm{ij}}+\tau_{\mathrm{i}}+\beta_{\mathrm{j}}++\varepsilon_{(\mathrm{ij})}$

Dónde: $\mathrm{i}=1,2$ y 3 tratamientos o sistemas de uso, $\mathrm{j}=1 \ldots 6$ fincas (en el paisaje), $\mathrm{k}=\mathrm{i} \ldots . .10$ fechas de monitoreo por cada gas. $\mathrm{Y}_{\mathrm{ijk}}=$ Es el valor repuesta del gas por fechas de muestreo tomadas en los distintos sistemas de uso de cada finca., $\mu=$ Efectos de las media general del modelo, $\tau_{\mathrm{i}}$ $=$ El efecto del tratamiento o uso del suelo, $\beta_{\mathrm{j}}=$ El efecto de la i-ésimo (finca), y Eijk = es el error de ( $\beta \theta \alpha)$ ijk. El nivel de significancia utilizado fue al 0.05 , comparada el efecto de los usos de la tierra con una prueba de LSD Fisher.

Para explicar la relación entre los factores temperatura y el WFPS con respecto al comportamiento de los gases $\mathrm{N}_{2} \mathrm{O}$ y $\mathrm{CH}_{4}$, se utilizó la técnica de Correlación Múltiple y el Análisis de Componentes Principales, utilizando 108 datos. Los análisis fueron corridos en InfoStat (2009), y graficados en SigmaPlot 12.0 versión libre. Los coeficientes de correlación se clasificaron de fuerte ( $>0.65$ a 1$)$, moderada (0.25 a 0.64) y débil (0.0 a 0.24) según (Kiernan, 2010). 2014.

\section{RESULTADOS Y DISCUSION}

Condiciones climáticas. La precipitación ocurrida de mayo a noviembre de 2014, registran un total de 68 eventos para la comunidad La Danta con un total de $1577 \mathrm{~mm}$ anuales. En la comunidad La Flor ocurrieron 55 eventos con un total de $1288 \mathrm{~mm}$ anuales. El mes con menor precipitación fue junio (40.4 y $50 \mathrm{~mm}$ ), y septiembre el de mayor precipitación (633 y $478.4 \mathrm{~mm}$ ), respectivamente en las comunidades $\mathrm{La}$ Danta y La Flor (Figura 4). Los pluviómetros fueron ubicados en la finca de la productora Teresa Ordoñez en la comunidad La Flor y en la casa del productor Roberto Pineda en la Comunidad La Danta.

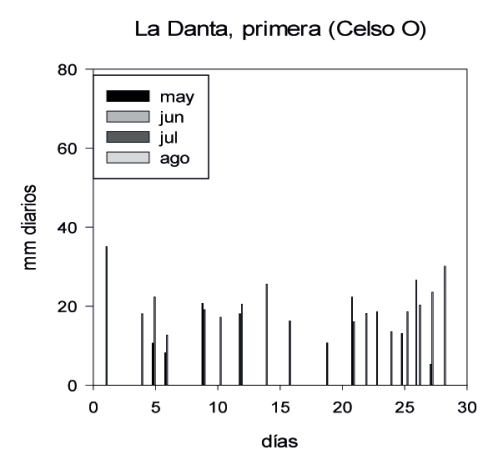

La Flor, primera (Teresa O)

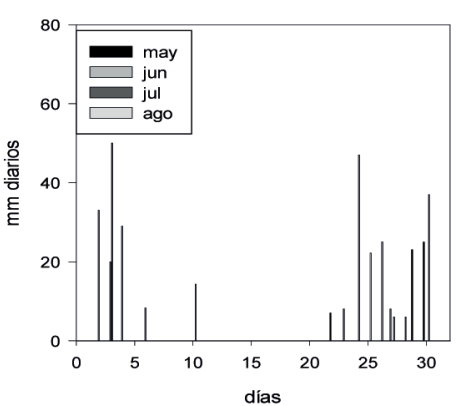

Figura 4. Precipitación diaria en los sitios experimentales. Proyecto CIAT-UNA GEI

Emisiones y flujos acumulados. Los flujos diarios de Metano $\left(\mathrm{CH}^{4}\right)$ fueron afectados significativamente por los tratamientos en los diferentes usos del suelo $(p>\mathrm{F}=0.0133)$, donde el sistema Quesungual fue sumidero y el tradicional emisor en algún momento del período de muestreo (Figura 5; Figura 7). Es posible que la variabilidad en los flujos de metano esté relacionada con los períodos secos y lluviosos (Ferreira, 2008).

El sistema Quesungual funcionó como sumidero con la excepción de las fincas de los productores Isidro Zúñiga y Teresa Rodríguez ( $40 \mathrm{mg} \mathrm{CH}_{4} \mathrm{~m}_{2} \mathrm{~d}^{-1}$ ) que presentaron valores positivos en cuanto a emisiones de metano en el mes de septiembre, mes donde más del $40 \%$ de las precipitaciones ocurrieron (Figura 5D y F). 
El sistema de siembra tradicional presentó picos de $\mathrm{CH}_{4}$ para el mes de noviembre a la salida de la estación lluviosa, en la finca del productor Freddy Aguilera $(15.28 \mathrm{mg}$ $\left.\mathrm{CH}_{4} \mathrm{~m}^{2} \mathrm{~d}^{-1}\right)$. En donde este gas alcanzó su máxima tasa de oxidación (Figura 5, B). En la misma finca durante el mes más lluvioso (septiembre), el sistema tradicional fue el mayor sumidero de $\mathrm{CH}_{4}$. Sin embargo, el bosque secundario mostró un flujo similar y bajo de emisión de metano en las seis fincas gobiernan la emisión del gas metano, y dependiendo de cuál predomine (metanogénesis o la metanotrofía), en el suelo puede producir que este funcione como sumidero o emisor del gas metano (Sedano et al., 2016). En el sistema tradicional es posible que predominara la actividad microbiana relacionada a la metanogenesis. de estudio (Figura 5). En el suelo dos procesos microbianos

Lemke y Wallander, 2008). Los procesos de desnitrificación que dan como resultado la producción de $\mathrm{N}_{2} \mathrm{O}$, ocurren en un WFPS mayor a $60 \%$, en condiciones de laboratorio (Linn \& Doran 1984).

En este estudio, los flujos de óxido nitroso presentaron gran variabilidad entre fechas de muestreo, sistemas de usos y entre fincas (Figura 6). En la temporada lluviosa la mayor emisión de $\mathrm{N}_{2} \mathrm{O}$ ocurrió en el sistema Quesungual (septiembre) coincidiendo con las fechas de fertilización (urea $46 \%$ ) del cultivo del frijol. Los valores promedios entre fincas fueron $\left(2.05 \mathrm{mg} \mathrm{N}_{2} \mathrm{O} \mathrm{m}^{-2} \mathrm{dia}^{-1}\right)$. Estas emisiones pueden estar vinculado a la humedad en el suelo y el WFPS, ya que en suelos arcilloso, la humedad puede haber logrado su punto de saturación al $49 \%$ de humedad (Fassbender, 1993) y así por efecto del WFPS aumentar el potencial de desnitrificación (Cuadro 3). Sin embargo, en la época seca, mayores emisiones de $\mathrm{N}_{2} \mathrm{O}$ ocurrieron a inicio de mayo, coincidiendo con la etapa de fertilización del cultivo de maíz (Figura 6; C y F), resultando la mayoría de las fincas ser emisoras de óxido nitroso; resultados que coinciden con los reportados en Lempira, Honduras por Ferreira, Rondón y Hurtado (2008).

En los sistemas de uso de suelo bosque secundario y sistema tradicional, los flujos de $\mathrm{N}_{2} \mathrm{O}$ fueron variables entre fechas de muestreo. Por ejemplo, en los sistemas tradicionales, la finca del señor Freddy Aguilera, presentó luego de la fertilización, el pico más alto de emisiones de $\mathrm{N}_{2} \mathrm{O}$ con

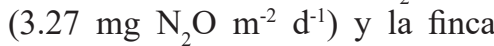
de Gerónimo Aguilera, alcanzó bajos niveles, funcionando como sumidero de $\mathrm{N}_{2} \mathrm{O}$ (Figura 6, B y C). En el bosque secundario para la época lluviosa, por ejemplo, las fincas de Freddy Aguilera, Isidro

Flujos diarios de óxido nitroso. Para este análisis se considera el suelo una fuente natural de este gas por estar relacionado al ciclo del nitrógeno, la aplicación de los fertilizantes, la descomposición de la materia orgánica, y el espacio poroso lleno de agua (WFPS). Lo que se relaciona con las emisiones de $\mathrm{N}_{2} \mathrm{O}$, dado que los suelos presentan mayor actividad biológica o respiración en un porcentaje cercano al $60 \%$ WFPS. Arriba del $60 \%$, la actividad microbiana se ve limitada por la reducción de la aeración y por debajo limitada por la disponibilidad de agua (Dusenbury, Engel, Miller,
Zúñiga y Juana Hernández mostraron que el sistema bosque funcionó como sumidero de $\mathrm{N}_{2} \mathrm{O}$ (Figura 6, B, D y E).

Flujos acumulados de $\mathrm{CH}_{4}$ y $\mathbf{N}_{2} \mathrm{O}$. Los flujos acumulados de metano fueron afectados significativa entre sistemas de uso ( $p>\mathrm{F}=0.0133)$, donde el sistema Quesungual funcionó como sumidero neto para el gas metano, y el bosque presentó bajas emisiones. Siendo el sistema tradicional, el único emisor, datos que coinciden con los valores presentados por Ferreira (2008). A nivel de productores las seis fincas lograron funcionar como sumidero de $\mathrm{CH}_{4}$ (Figura 7). 

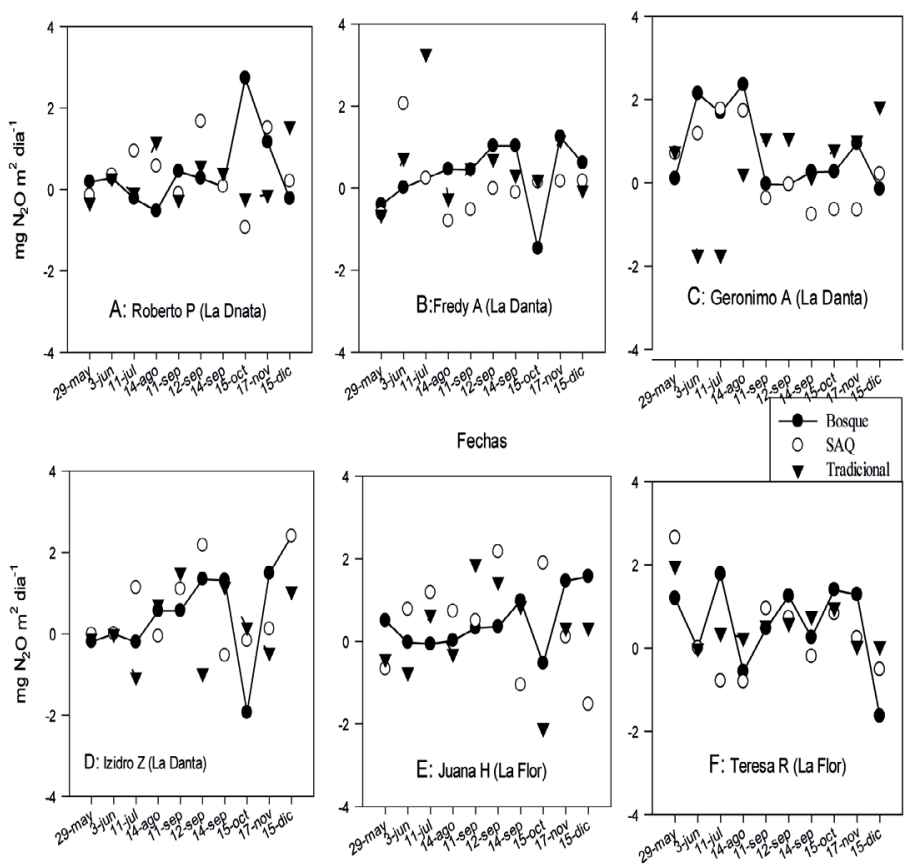

Fechas

Figura 6. Flujos diarios en $\mathrm{mg} \mathrm{N}_{2} \mathrm{O} \mathrm{m}^{2}$ dia $^{-1}$ por finca de productores. Somotillo, Chinandega 2014. Proyecto CIAT-UNA GEI 2014.

Finalmente, los flujos acumulados de emisiones de $\mathrm{N}_{2} \mathrm{O}$ fueron mayores en los sistemas tradicionales, observándose menores emisiones en los sistemas Quesungual y Bosque. A nivel de fincas los productores Juana Hernández y Roberto Pineda obtuvieron los valores más altos en flujos acumulados de $\mathrm{N}_{2} \mathrm{O}$ y con menor cantidad, la finca de la productora Teresa Rodríguez, donde también se observa mayor flujo acumulado de $\mathrm{NH}_{4}$ (Figura 7 y 8).

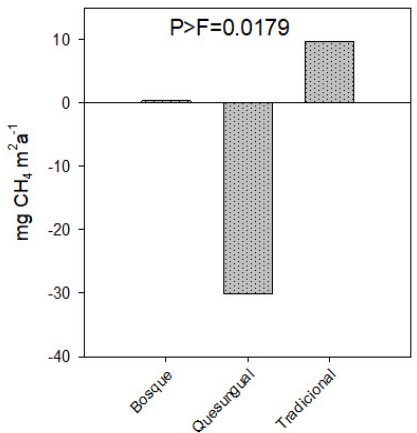

Sistema de uso de suelo

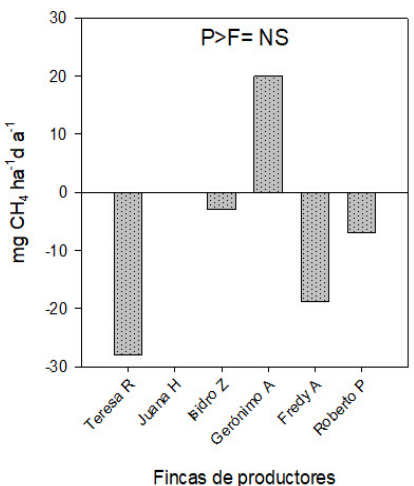

Figura 7. Flujos acumulados en $\mathrm{mg} \mathrm{CH}_{4} \mathrm{~m}^{2} \mathrm{a}^{-1}$ a nivel de sistemas de uso del suelo y fincas. Somotillo, Chinandega de mayo a diciembre, 2014. Proyecto CIAT-UNA GEI 2014.

El análisis de emisiones para el gas metano $\left(\mathrm{CH}_{4}\right)$ indica que los sistemas tradicionales con menor cobertura vegetal ofrecen mayores emisiones de gases metano, y el sistema agroforestales Quesungual se convierte en sumidero de este gas.

El análisis de correlación muestra asociaciones positivas entre las emisiones de los gases $\mathrm{NH}_{4}$ y $\mathrm{N}_{2} \mathrm{O},\left(\mathrm{r}^{2}=1\right.$; $p>\mathrm{F}=0.0533$ ), y tendencias negativas para la asociación entre el WFPS, y el incremento de las temperaturas en la superficie del suelo, similar con las emisiones de $\mathrm{NH}_{4} \mathrm{y}$ $\mathrm{N}_{2} \mathrm{O}$. Los valores de WFPS fueron menores en el sistema tradicional (48\%), y mayores en sistema Quesungual y bosque (53\%). Comportamiento inverso se observó en las temperaturas en el suelo, donde menores temperaturas las presentó el sistema bosque y Quesungual (entre 28 y $32{ }^{\circ} \mathrm{C}$ ), y más caliente en el sistema Tradicional con 35.5 ${ }^{\circ} \mathrm{C}$ promedio, durante el monitoreo realizado antes de las 12:00 m (Cuadro 2 y 3).

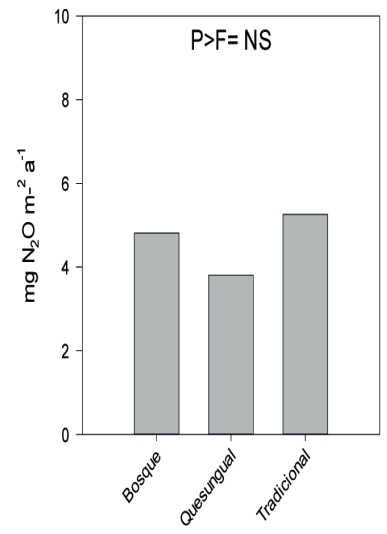

Sistemas de uso del suelo

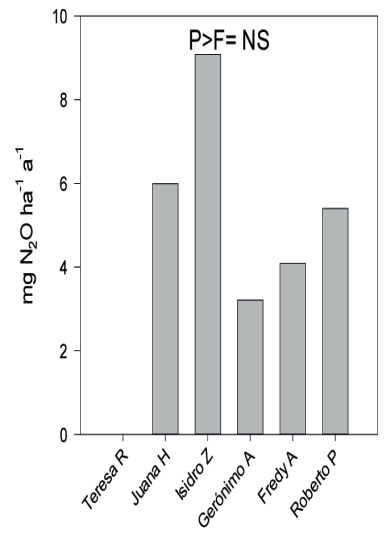

Fincas de productores
Figura 8. Flujos acumulados en $\mathrm{mg} \mathrm{N}_{2} \mathrm{O} \mathrm{m} \mathrm{m}^{-2} \mathrm{a}^{-1}$ a nivel de sistemas de uso del suelo y fincas. Somotillo, Chinandega, de mayo a diciembre, 2014. Proyecto CIAT-UNA GEI 2014.

Cuadro 2. Correlación múltiple entre flujos promedio de gases de efecto invernadero y las variables WFPS.

\begin{tabular}{lllll}
\hline & Temperatura & $\mathrm{CH}_{4}$ & $\mathrm{~N}_{2} \mathrm{O}$ & WFPS \\
\hline Temperatura & 1.00 & & & \\
$\mathrm{CH}_{4}$ & $0.23 \mathrm{NS}$ & 1.00 & & \\
$\mathrm{~N}_{2} \mathrm{O}$ & $0.31 \mathrm{NS}$ & 1.00 & & \\
& & 0.0533 & 1.00 & \\
WFPS & $-0.90 \mathrm{NS}$ & -0.63 & -0.70 & \multirow{2}{*}{1.00} \\
& & $\mathrm{NS}$ & $\mathrm{NS}$ & \\
\hline
\end{tabular}

La fila en el valor superior representa el coeficiente de correlación y el valor en la parte baja la probabilidad 
Cuadro 3. Temperatura superficial del suelo dentro de las cámaras, WFPS, $\mathrm{CH}_{4}$ y $\mathrm{N}_{2} \mathrm{O}$, por sistema de uso de suelos, 2014.

\begin{tabular}{|c|c|c|c|c|c|}
\hline Tratamiento & Variable & $\mathrm{n}$ & Media & $\begin{array}{l}\text { Desviación } \\
\text { Estándar }\end{array}$ & $\begin{array}{l}\text { Coeficiente } \\
\text { de } \\
\text { Variación }\end{array}$ \\
\hline Bosque & Temperatura & 36 & 27.28 & 5.27 & 18.83 \\
\hline Bosque & $\mathrm{CH}_{4}$ & 36 & 0.34 & 47.60 & 13830.04 \\
\hline Bosque & $\mathrm{N}_{2} \mathrm{O}$ & 36 & 4.81 & 18.84 & 392.12 \\
\hline Bosque & WFPS & 36 & 53.17 & 16.68 & 31.37 \\
\hline Quesungual & Temperatura & 36 & 31.71 & 4.95 & 15.60 \\
\hline Quesungual & $\mathrm{CH}_{4}$ & 36 & $\begin{array}{l}- \\
30.08\end{array}$ & 66.56 & 221.30 \\
\hline Quesungual & $\mathrm{N}_{2} \mathrm{O}$ & 36 & 3.81 & 15.60 & 409.14 \\
\hline Quesungual & WFPS & 36 & 52.80 & 19.21 & 36.38 \\
\hline Tradicional & Temperatura & 36 & 35.52 & 3.99 & 11.22 \\
\hline Tradicional & $\mathrm{CH}_{4}$ & 36 & 9.77 & 61.06 & 625.04 \\
\hline Tradicional & $\mathrm{N}_{2} \mathrm{O}$ & 36 & 5.26 & 14.29 & 171.75 \\
\hline Tradicional & WFPS & 36 & 48.12 & 20.32 & 42.24 \\
\hline
\end{tabular}

\section{CONCLUSIONES}

Las emisiones del gas metano $\left(\mathrm{CH}_{4}\right)$ observadas únicamente en el sistema tradicional de siembra, y la fuerte asociación positiva al incremento de las temperaturas por la ausencia de árboles, explica el rol de la temperatura para expandir o contraer el gas en el suelo. Fenómeno inverso al flujo acumulado $\mathrm{N}_{2} \mathrm{O}$, que presenta emisiones mayores en sistemas con presencia de árboles (Bosque y Quesungual), un gas que resulta emisor donde las temperaturas tienden a ser menores. Fenómeno asociado a los altos contenidos de biomasa superficial y la fuerte actividad biológica.

La asociación esperada entre el flujo del óxido nitroso $\left(\mathrm{N}_{2} \mathrm{O}\right)$ y el porcentaje de poros saturados con agua (WFPS) no se pudo observar en campo, lo que podría explicar, que dicha relación está afectada por otras variables como la respiración radicular, el tipo de muestreo y la variación de las propiedades físicas y químicas de los suelos en campo.

\section{REFERENCIAS BIBLIOGRÁFICAS}

Arias-Navarro, C., Díaz-Pinés, E., Kiese, R., Rosenstock, T. S., Rufino, M. C., Stern, D.,... Butterbach-Bahl, K. (2013). Gas pooling: A sampling technique to overcome spatial heterogeneity of soil carbon dioxide and nitrous oxide fluxes. Soil Biology and Biochemistry, 67, 20-23. http://doi.org/10.1016/j.soilbio.2013.08.011

Avilés, S; Esteban, A. (2016). Efectos de los sistemas de cultivo - arboles - pasto sobre la erosión laminar y calidad de suelo en la microcuenca Tecomapa, municipio de Somotillo- Nicaragua. (Tesis de maestría). Universidad Nacional Agraria, Managua.

Bremner, J.M. and Mulvaney, C.S. (1982) Nitrogen-Total. In: Methods of soil analysis. Part 2. Chemical and microbiological properties. Page, A.L., Miller, R.H. and Keeney, D.R. Eds., American Society of Agronomy, Soil Science Society of America, Madison, Wisconsin, 595-624.

Dusenbury, M. P., Engel, R. E., Miller, P. R., Lemke, R. L., \& Wallander, R. (2008). Nitrous oxide emissions from a northern great plains soil as influenced by nitrogen management and cropping systems. Journal of environmental quality, 37(2), 542-550.

Fassbender; HW. (1993). Modelos edafológicos de sistemas agroforestales. CATIE Turrialba, Costa Rica.

Ferreira Catrileo, OI. (2008). Flujo de Gases de efecto Invernadero, Potencial de Calentamiento Global y Evaluación de Emergía del Sistema Agroforestal Quesungual en el Sur De Lempira, Honduras. (Tesis de maestría). Palmira, CO.

Ferreira, OI. Rondón, MA; Hurtado, MP. (2008). Flujos de Gases de Efecto Invernadero Del Sistema Agroforestal Quesungual, Sistema de Tala Y Quema Y del Bosque Secundario en el Sur de Lempira, Honduras. Recuperado de https://cgspace.cgiar.org/bitstream/ handle/10568/21825/21825.pdf?sequence $=1 \&$ is Allowed $=y$

InfoStat (versión 2009). [Software computacional de estadística]. Universidad Nacional de Córdoba, Argentina.

Instituto Nicaragüense de Estudios Territoriales. (2014). Resumen Meteorológico anual del período 1958 al 2014. Managua, Nicaragua: Dirección de Meteorología.

Instituto Nicaragüense de Tecnología Agropecuaria (2005). Diagnóstico sub cuenca Tecomapa. Chinandega, Nicaragua: Oficina de extensión Somotillo Pacífico norte.

Kiernan, D.H. (2010). Introductory Statistics for Environmental Sciencies. Lecture Supplement and Workbook. Kendall Hunt, publishing company. Dubuque, IA. 223p.

Khatun K., Imbach P, Zamora JC (2013). The implications of climate impacts on conservation strategies for central America using the Holdridge Life Zone (HLZ) land classification. Forest 6: 183-189. Recuperado de https://www.siswf.it/iforest/ contents/?id=ifor0743-006.

Linn, MD; Doran, JW. 1984. Effect of Water -Filled Pore Space on Carbon Dioxide and Nitrous Oxide Production in Tilled and Nontilled Soil. (En línea). Soil society American Journal. 1267-1272 p. consultado 7 mayo 2016. Disponible en http://naldc.nal. usda.gov/download/16745/PDF

Nair, PKR; Nair, VD; Kumar, BM; Haile, SG. (2009). Soil carbon sequestration in tropical agroforestry systems: a feasibility appraisal. Environmental Science \& Policy, 10: 10-16.

Organización de las Naciones Unidas para la Alimentación y la Agricultura. (2019). Latinoamérica duplicó sus emisiones agrícolas de gases de efecto invernadero en los últimos 50 años. FAO. Recuperado de http://www.fao.org/americas/noticias/ver/es/c/240450/ 
Programa de las Naciones Unidas para el Desarrollo en Nicaragua. (2008). Documento de Proyecto. Programa de pequeñas donaciones. Recuperado de https://info.undp.org/docs/pdc/Documents/NIC/00034097_Prodoc\%2000036000.pdf

Rodríguez, I. I. Calero, A. Avilés, A. Mendoza, R. B. (2008). Estudio detallado de suelos en Área de laderas por toposecuencia de la Microcuenca la Danta, Somotillo - Chinandega (Informe). Universidad Nacional Agraria. Managua - Nicaragua.

Sedano, F., Silva, J. A., Machoco, R., Meque, C. H., Sitoe, A., Ribeiro, N., ... \& Tucker, C. J. (2016). The impact of charcoal production on forest degradation: a case study in Tete, Mozambique. Environmental Research Letters, 11(9), 094020. Recuperado de http:// www.iopscience.iop.org/article/10.1088/1748-9326/11/9/094020 